\title{
Processus de création dans le théâtre de marionnettes : présentation d'un dossier génétique
}

\section{Cristiane Miryam Drumond de Brito}

Traducteur : Maria Ignez Mena Barreto

\section{OpenEdition \\ Journals}

Édition électronique

URL : http://journals.openedition.org/genesis/553

DOI : 10.4000/genesis.553

ISSN : 2268-1590

\section{Éditeur :}

Presses universitaires de Paris Sorbonne (PUPS), Société internationale de génétique artistique littéraire et scientifique (SIGALES)

\section{Édition imprimée}

Date de publication : 1 janvier 2011

Pagination : 219-227

ISBN : 978-2-84050-749-9

ISSN : $1167-5101$

Référence électronique

Cristiane Miryam Drumond de Brito, «Processus de création dans le théâtre de marionnettes :

présentation d'un dossier génétique », Genesis [En ligne], 32 | 2011, mis en ligne le 18 septembre 2012, consulté le 30 avril 2019. URL : http://journals.openedition.org/genesis/553 ; DOI : 10.4000/ genesis.553 


\title{
Processus de création dans le théâtre de marionnettes : présentation d'un dossier génétique
}

\author{
Cristiane Miryam Drumond de Brito*
}

I

l est un processus de création sur lequel la critique génétique n'a pas encore songé à se pencher : celui qui aboutit à un spectacle de marionnettes. Il s'agit pourtant d'un domaine très riche, qui met en jeu un grand nombre de formes d'expression. Les arts plastiques y occupent une place importante et jouent un rôle essentiel dans la construction des personnages. À l'instar du théâtre, et plus encore que le théâtre, cet art de la performance appelle une "génétique qui englobe non seulement l'avant-texte, mais aussi "l'avant-scène", c'est-à-dire tout ce qui porte trace de la préparation du spectacle ${ }^{1} »$. À titre préliminaire, nous ferons ici une rapide présentation, sans commentaires, des éléments qui composent le dossier génétique d'un spectacle particulièrement innovant.

Le Journal ( $O$ Diario), spectacle de marionnettes d'Álvaro Apocalypse, a été mis en scène en trois différentes versions : la première, à l'occasion du Festival d'hiver de l'Université fédérale du Minas Gerais (UFMG) à Belo Horizonte (Brésil) sous le titre Diario de um tímido forasteiro (Journal d'un outsider timide) (1990) ; la deuxième, à l'occasion du Festival du son et du mouvement, en France (1992) et la troisième, devant un public élargi à Rio de Janeiro (1997). Malgré les transformations qui ont eu lieu d'une présentation à l'autre (type de manipulation des marionnettes, personnages, éclairage, musique, manière de concevoir les scènes, dialogues, etc.), on peut considérer qu'il s'agit d'un seul projet poétique en métamorphose.

Álvaro Apocalypse, artiste plasticien né à Ouro Fino (Minas Gerais) a dessiné depuis l'enfance. Cette pratique lui a valu d'être professeur de dessin et dans cette fonction il a travaillé sur la figure humaine, son anatomie et son inscription dans la culture, à travers la capoeira, les danses, fêtes et autres mythes populaires. Il a été également professeur et directeur de la faculté des Beauxarts de l'Université fédérale du Minas Gerais.

En 1970, Álvaro Apocalypse, son épouse et une amie créent la troupe de théâtre de marionnettes Giramundo : née d'un groupe d'artistes plasticiens, les arts plastiques y jouent un rôle important. Álvaro Apocalypse lui-même se considérait comme un artiste plasticien travaillant pour les arts de la scène. À la tête de Giramundo, il crée plusieurs spectacles, parmi lesquels les trois versions du Journal dont il est question ici.

Dès sa création, Álvaro Apocalypse et la troupe Giramundo ont privilégié la recherche dans le domaine de la dramaturgie et des différents langages que comprend le théâtre de marionnettes, ajoutant de nouveaux auteurs, de nouveaux thèmes et de nouvelles problématiques au répertoire du théâtre de marionnettes brésilien. Ont été aussi introduites des thématiques destinées à un public adulte : le théâtre de marionnettes n'est désormais plus l'apanage des enfants. Dans les documents de genèse présentés ici, on observe la recherche de formes poétiques déployées dans le dialogue avec l'univers culturel des adultes.

\footnotetext{
* Je tiens à rendre hommage à Álvaro Apocalypse, qui a bien voulu me confier ses manuscrits; et à remercier Cecilia A. Salles pour m'avoir guidée dans mon travail doctoral sur le processus de création d' Álvaro Apocalypse.

1. Almuth Grésillon et Nathalie Léger, «Brouillons de l'éphémère. Pour une génétique du théâtre », Genesis, n 26, «Théâtre », 2005, p. 7.
} 
Le dossier comprend des types de documents très divers :

1) Scénarios diagrammatiques

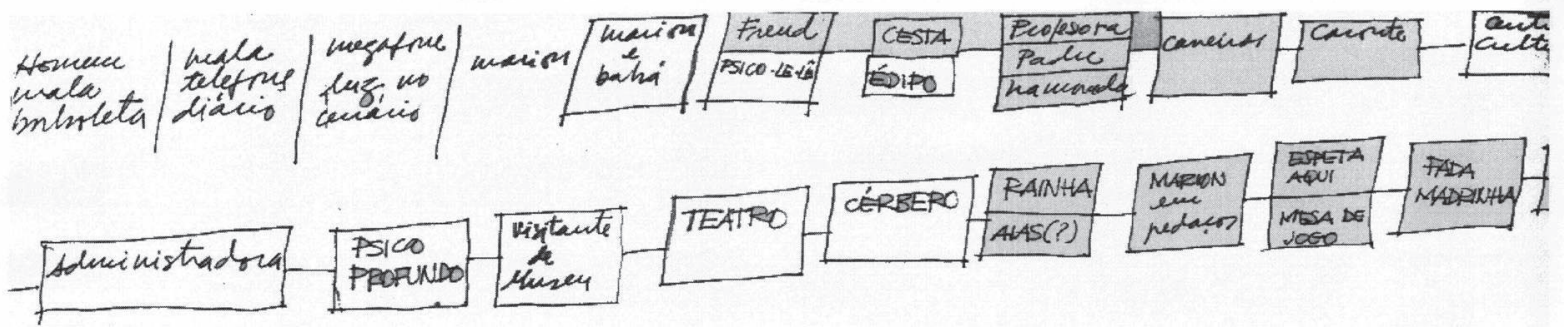

Fig. 1 : Schéma figurant les personnages dans leur ordre d'entrée en scène

2) Scénarios visuels présentant des séquences narratives

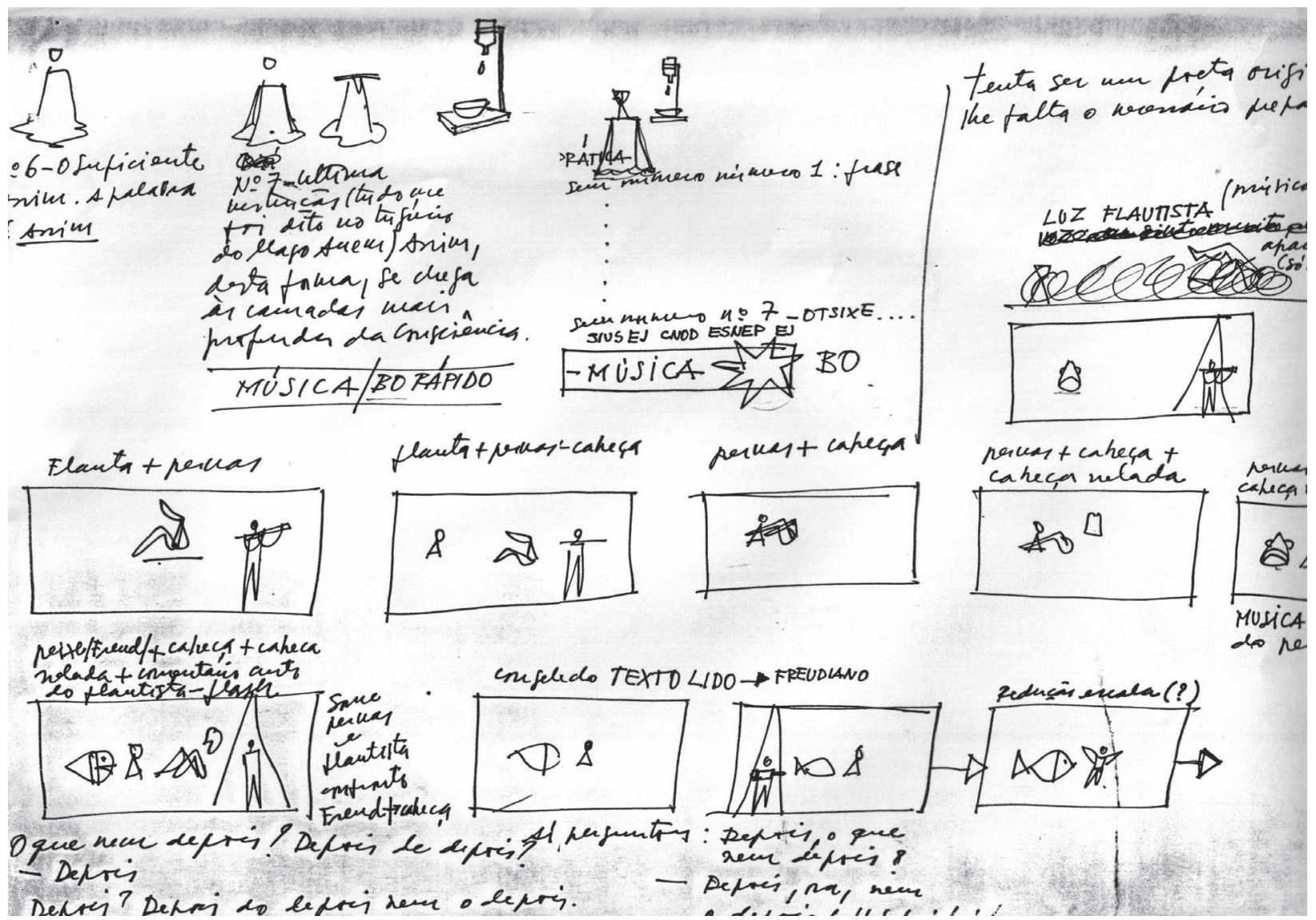

Fig. 2 : Croquis articulant le texte et la mise en scène 
3) Scénarios textuels

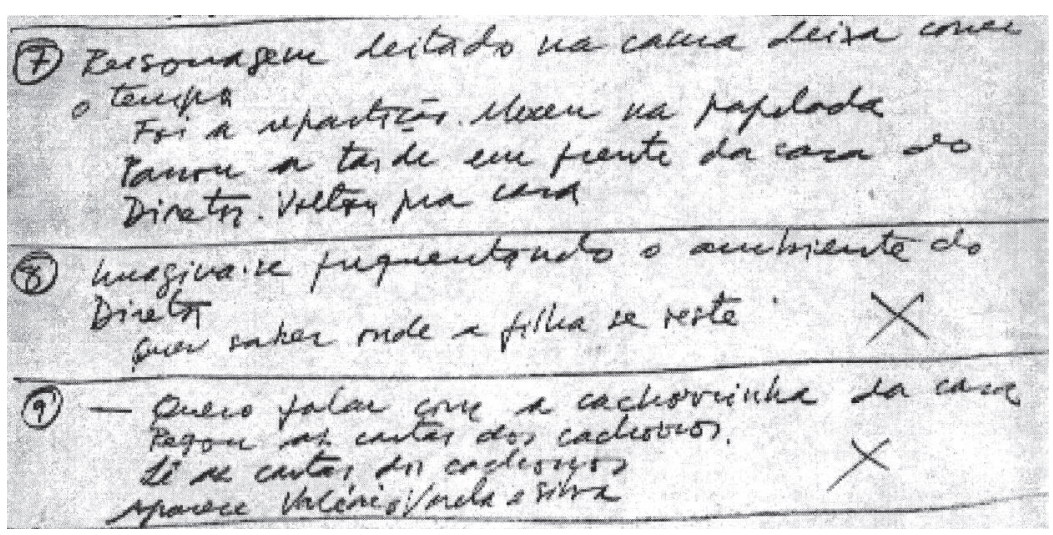

[Fig. 3 : traduction

7) Personnage couché sur un lit laisse passer le temps Il est allé au bureau. A tripoté de la paperasse.

A passé l'après-midi devant la maison du

Directeur. Est rentré à la maison

8) Il s'imagine chez le Directeur

Veut savoir où la fille s'habille

9) Je veux parler avec la petite chienne de la maison

Il a pris les lettres des chiens

Lit les lettres des chiens

Valerio Varela e Silva apparaît

4) Scénarios de répétition

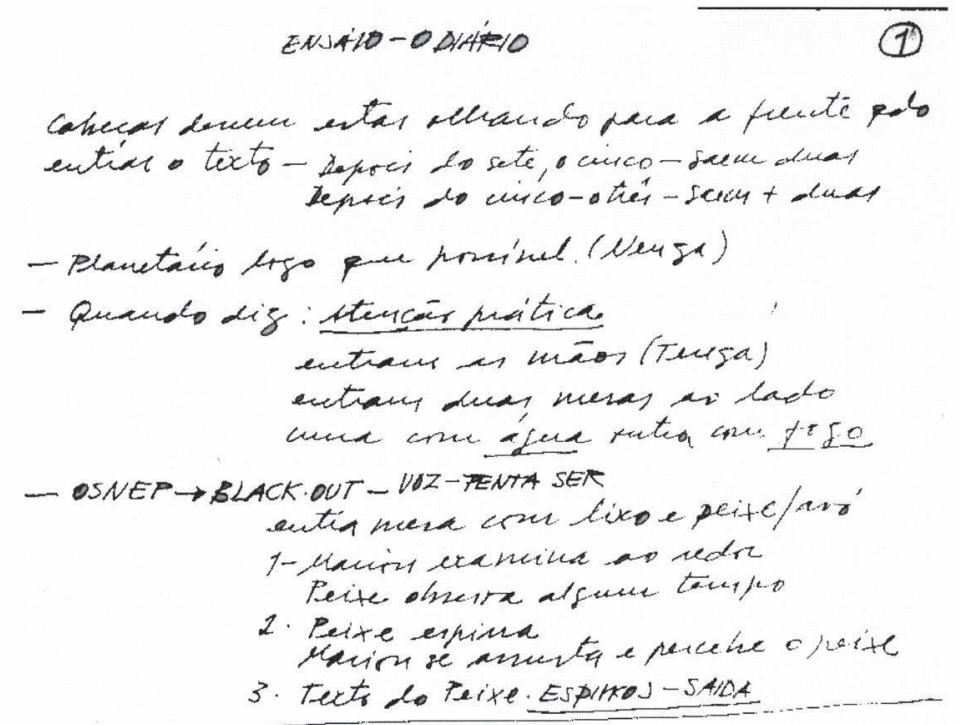

Fig. 4 : Consignes pour la mise en scène lors des répétitions 
5) Différentes versions des textes dramaturgiques, que j'appellerai version A, B, C, etc.

Extrait de la version A

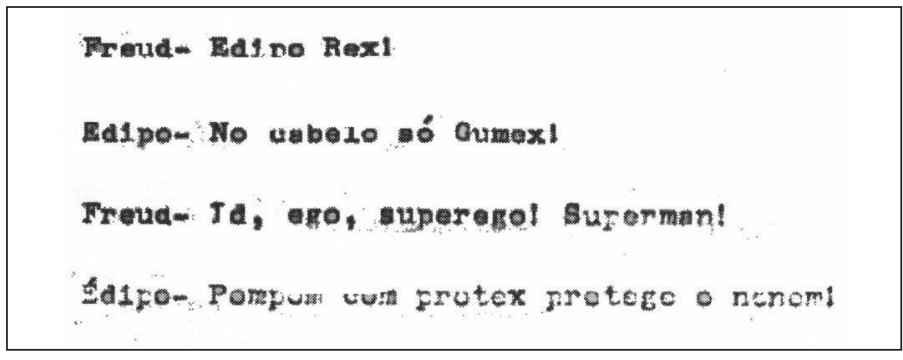

Fig. 5

Extrait de la version $B$

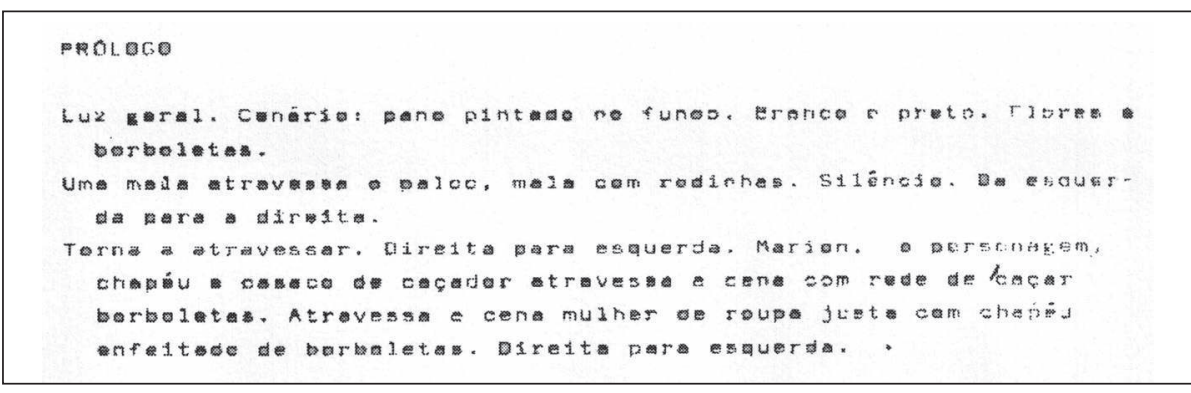

Fig. 6

6) Annotations au sujet des personnages et de leur voix

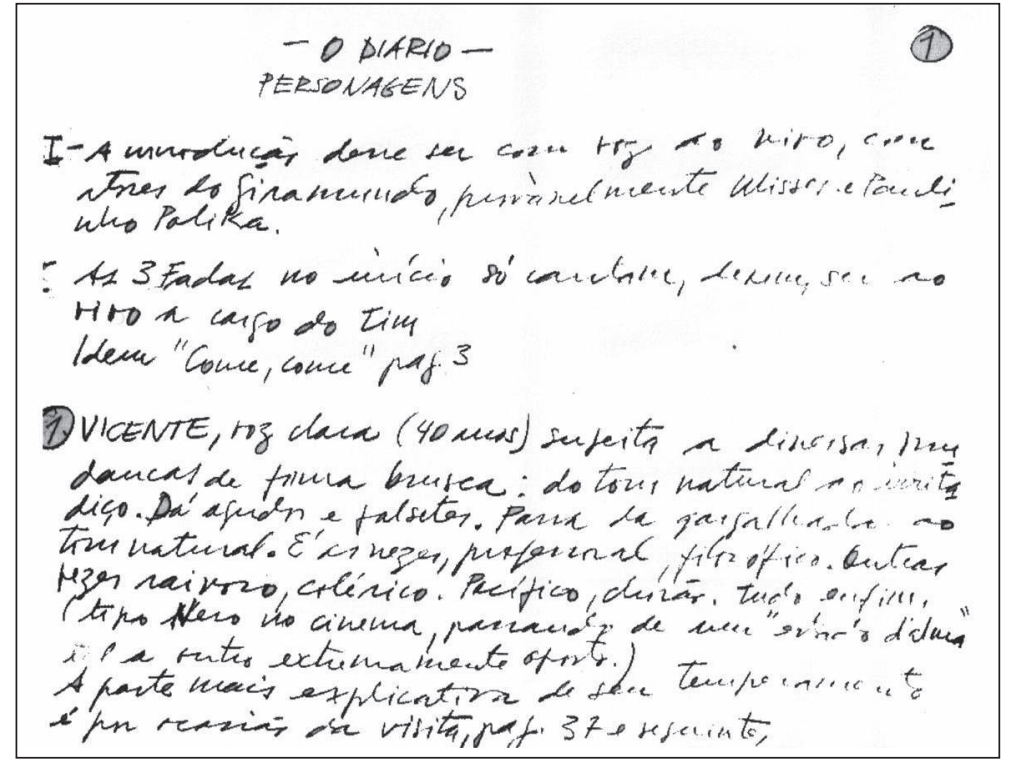

Fig. 7 :

- Indications concernant la voix off

- Indications concernant la voix des trois fées

- Caractérisation du personnage vinCENT à travers la description très précise du grain, de la tonalité et des inflexions caractéristiques de sa voix 
7) Ébauches des marionnettes

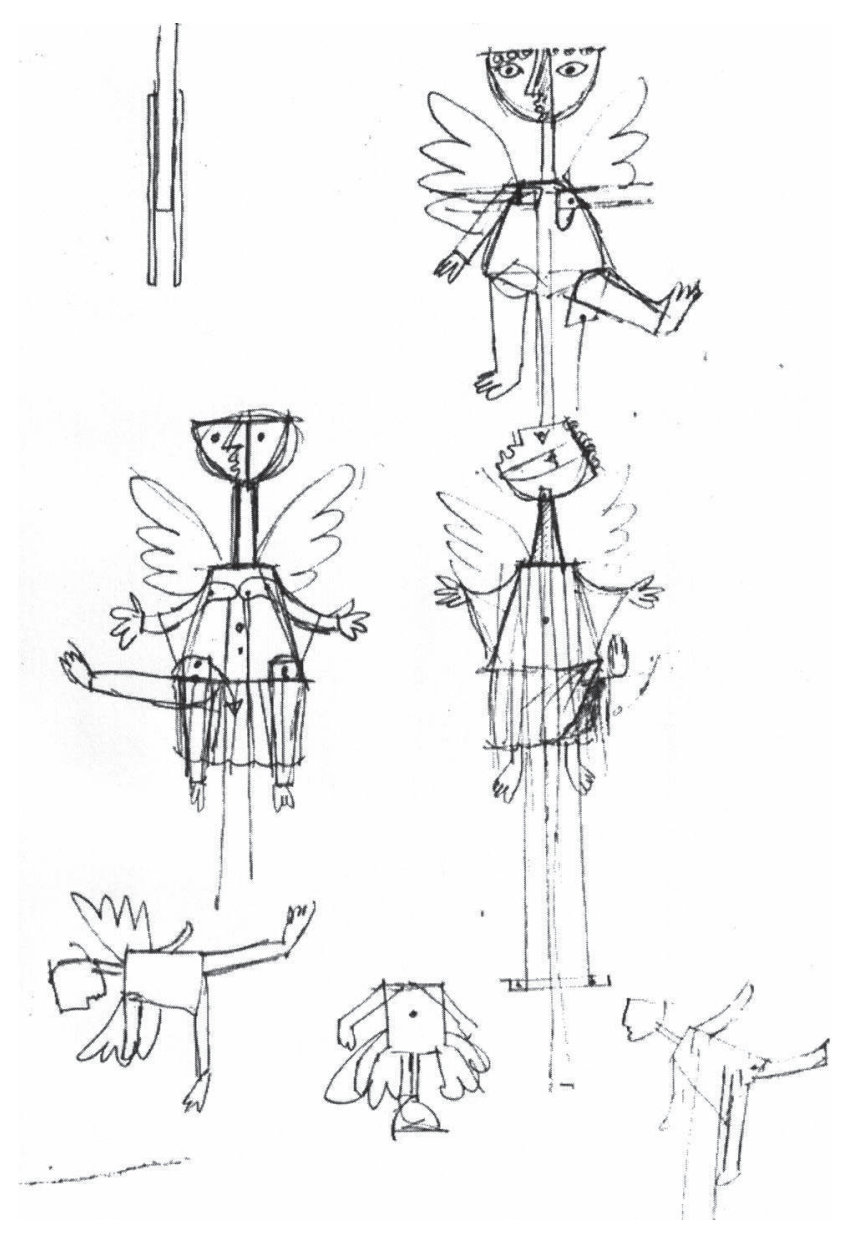

Fig. 8
8) Études de manipulation

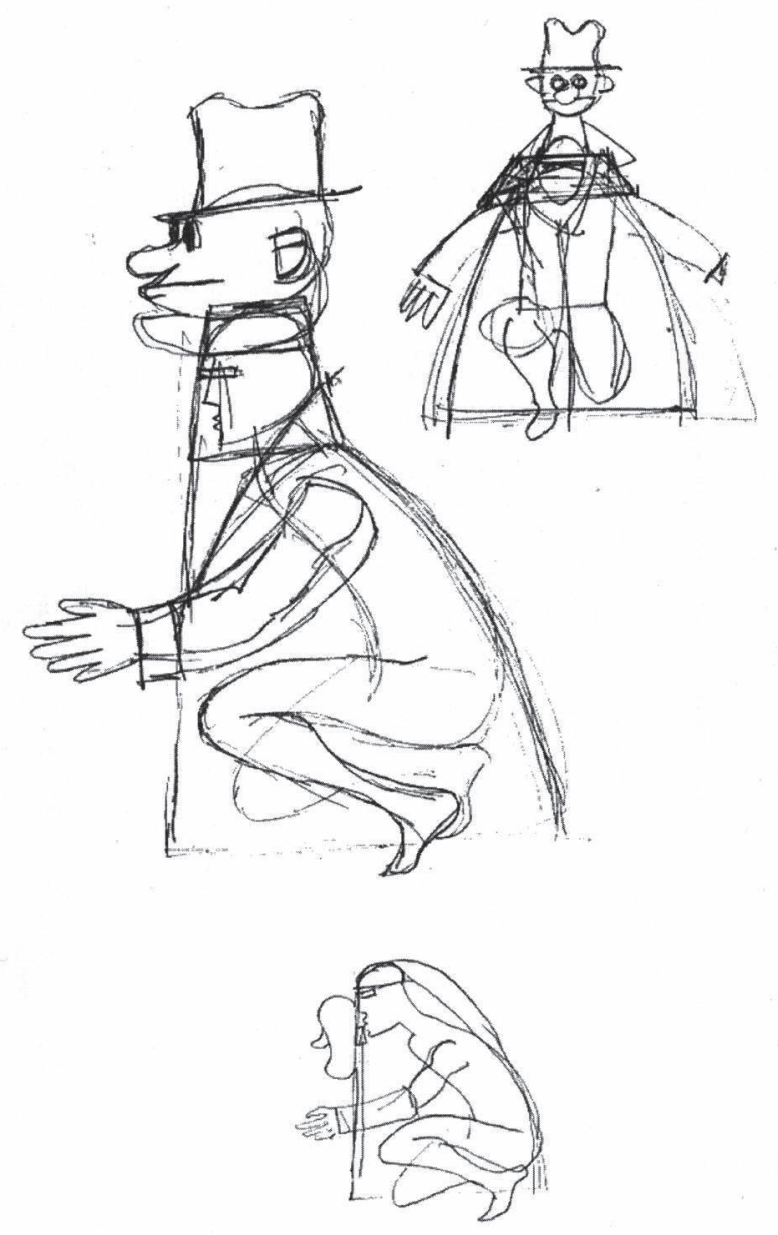

Fig. 9 
9) Ébauches de décor
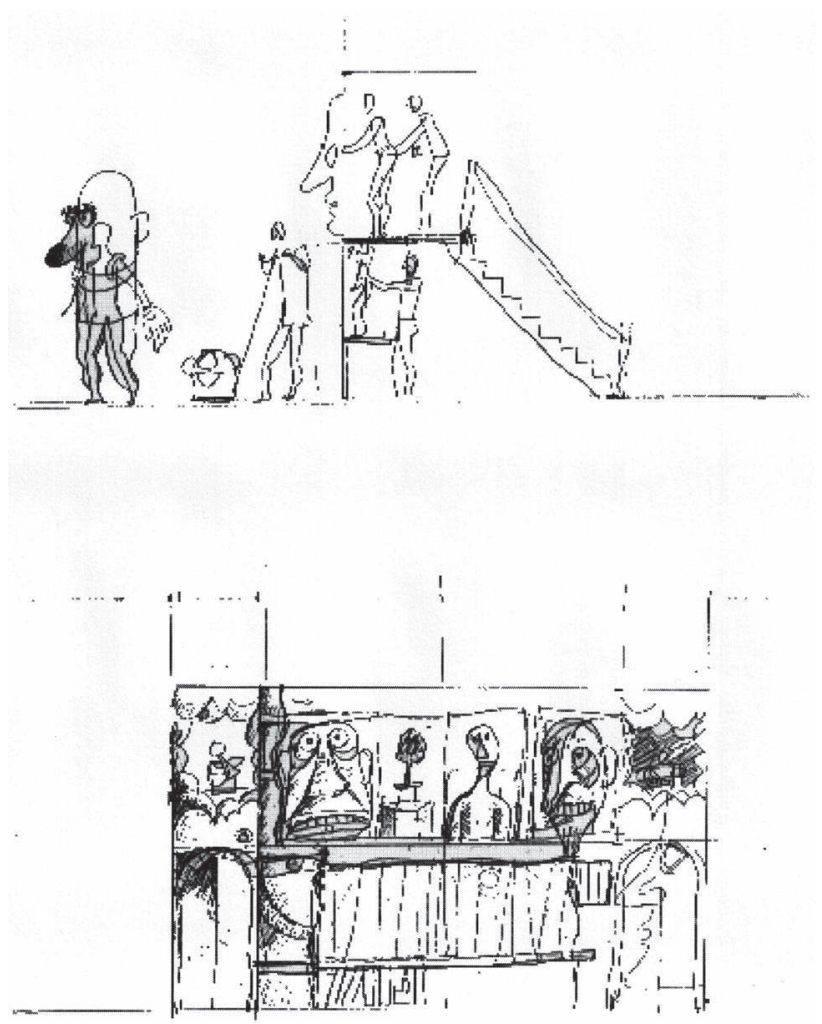

Fig. 10
10) Photos
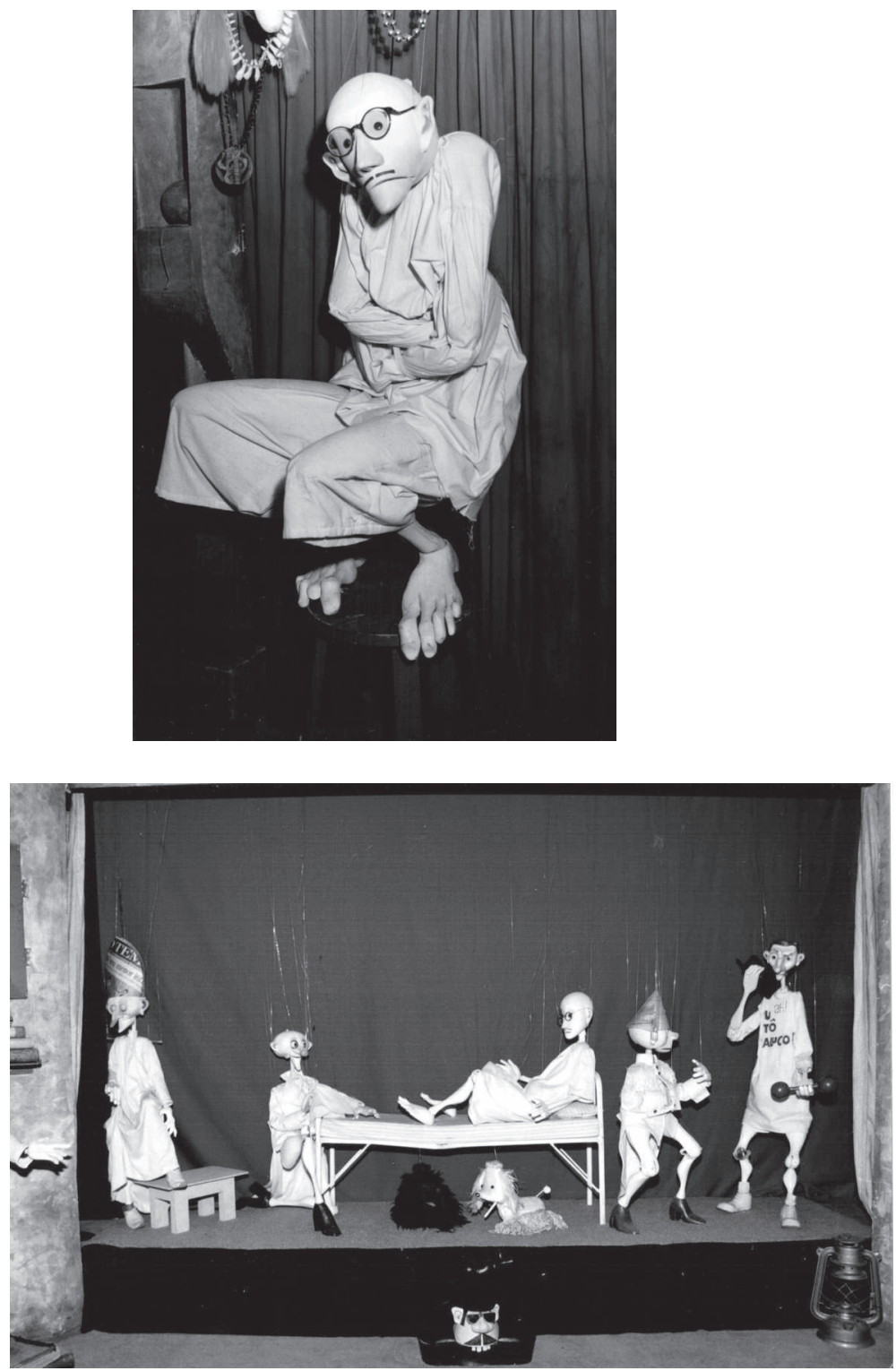

Fig. 11 et 12 
Le théâtre a une nature collective, comme l'attestent les documents eux-mêmes, mais la présence d'Álvaro Apocalypse à la tête de l'équipe est une constante. L'auteur se soucie de tout, depuis la conception d'un personnage jusqu'à l'éclairage, en passant par la musique, les tonalités des voix des marionnettes, etc. Dans ces papiers, nous retrouvons également des réflexions théoriques et des annotations au sujet du symbolisme associé aux marionnettes :

a)

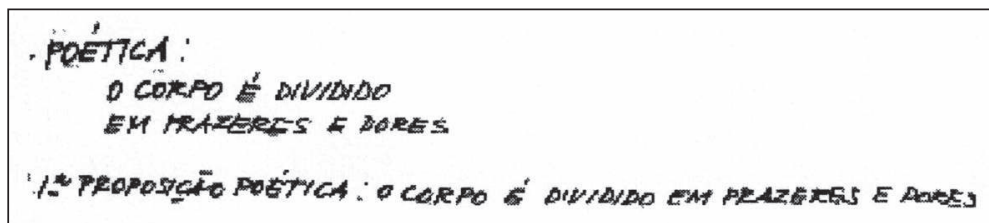

[Fig. 13 : traduction du document :

Poétique :

Le corps est divisé

En plaisir et douleur

1re proposition poétique : le corps est divisé en plaisirs et douleurs]

b)

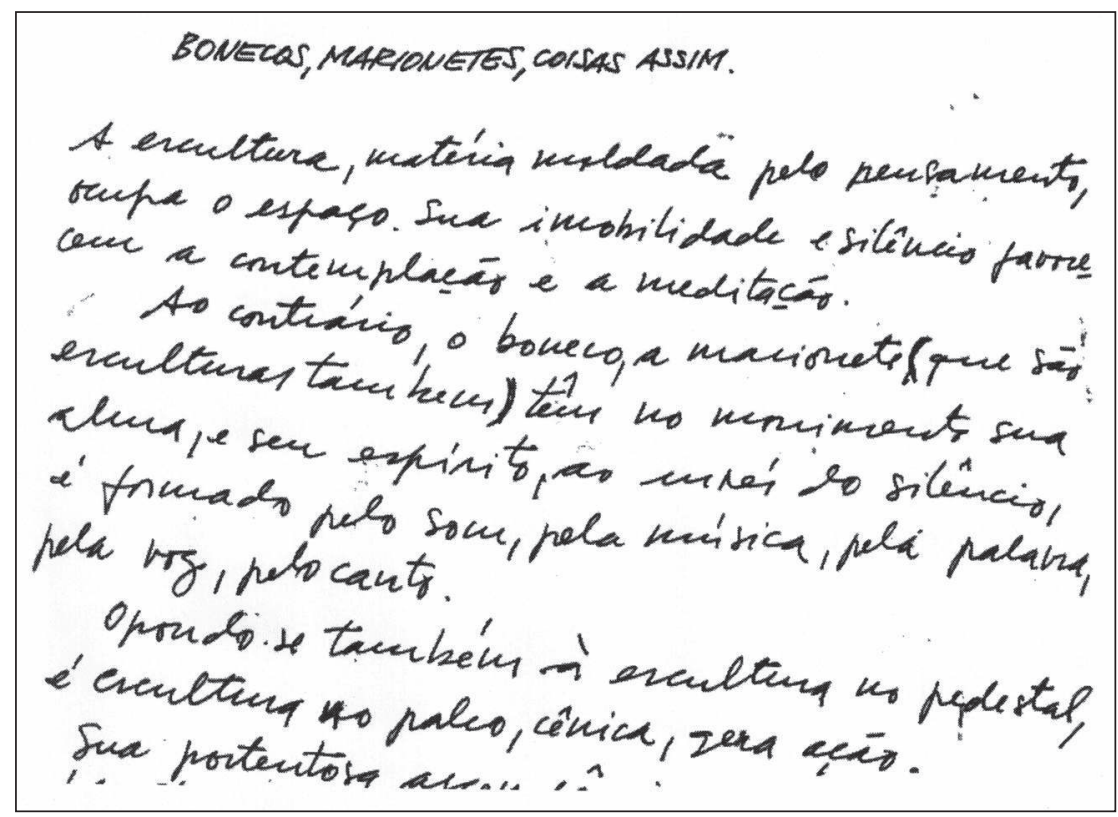

[Fig. 14 : traduction du document :

POUPÉES, MARIONNETTES, DES CHOSES COMME ÇA.

La sculpture, matière modelée par la pensée, remplit l'espace. Son immobilité et son silence favorisent la contemplation et la méditation.

Au contraire, la poupée, la marionnette (qui sont des sculptures aussi) ont dans le mouvement leur âme ; et leur esprit est formé non pas par le silence, mais par le son, par la musique, par la parole, par la voix, par le chant.

En s'opposant aussi à la sculpture sur son socle, elle est sculpture sur scène, théâtrale, elle génère de l'action. 
Outre les documents que nous avons présentés, on trouve des plans de scène en élévation, avec vue frontale et vue latérale, des plans d'éclairage, des paroles de chansons. Nous avons eu accès à des projets de marionnettes au format $68 \times 95 \mathrm{~cm}$, à des e-mails échangés entre Álvaro Apocalypse, des amis et la productrice. On peut également consulter des dossiers de presse rédigés par Álvaro Apocalypse, des entretiens, des programmes de pièces présentées et le Journal d'un fou de Gogol2.

L'œuvre d'Álvaro Apocalypse intègre de nombreux éléments de la culture brésilienne. En tant que marionnettiste, il travaille des images dans un pays ayant une riche tradition d'images et de couleurs, dans l'art sacré du baroque, dans les fêtes populaires, dans l'architecture...

Il faut également tenir compte des souvenirs d'enfance qu'Álvaro Apocalypse évoque dans son autobiographie, les jouets ramassés dans la nature ou construits par lui et ses frères, le dessin d'anatomie, très tôt enseigné par son père médecin. Cette expérience de l'enfance est transposée dans son travail de marionnettiste, qui met le fantastique, l'imagination, l'étude de l'anatomie et du mouvement au service de ses desseins, tels qu'on peut les percevoir dans les documents présentés ici :

- poser les bases d'une mythographie brésilienne ;

- établir des correspondances entre les langages littéraire, musical, pictural... en créant un jeu de scène et non uniquement un théâtre de marionnettes ;

- élaborer un nouveau concept esthétique et éthique pour le théâtre de marionnettes, en élargissant la recherche, au-delà du seul théâtre de marionnettes, à d'autres mouvements artistiques contemporains.

\section{Traduit du brésilien par Maria Ignez Mena Barreto}

2. Dans ses écrits, Álvaro Apocalypse note le statut ambivalent de son spectacle par rapport à l'ouvrage de Gogol : «Le Journal est une création originale. Originale, car au premier montage, Giramundo utilisait des scènes déjà conçues et n'avait pas de texte. Jusqu'à la rencontre avec le Journal d' un fou. Le Journal d' un fou est un conte de Gogol fréquemment adapté pour le théâtre, la plupart du temps sous forme de monologue. Giramundo l'a adapté d'une tout autre façon : les personnages cités par le narrateur ont été créés matériellement et participent de l'action en donnant la réplique au protagoniste. De telle sorte que le récit a été pratiquement éliminé de la pièce. D'où l'idée que ce montage n'est pas, à proprement parler, une adaptation du Journal d'un fou, car une grande part du matériau utilisé préexistait au montage et ne provenait pas de l'original de Gogol. » 
Cristiane Myriam Drumond de Brito est professeur d'ergothérapie à l'université fédérale de São Carlos (état de São Paulo). Elle a mené une recherche sur le processus créatif de l'auteur et marionnettiste Álvaro Apocalypse. Son ouvrage Rascunhos da Loucura («Brouillons de la folie », Editora Univap, 2001) traite de l'interaction des études de critique génétique avec d'autres champs de la connaissance. Actuellement, elle coordonne un projet de recherche et de diffusion qui élargit le processus de création chorégraphique au-delà du directeur de la compagnie et de ses danseurs et s'efforce d'établir dialogues et récits sur une base communautaire, en utilisant la critique génétique comme outil de construction du spectacle.

Cristiane M. Drumond de Brito, cdru@uol.com.br

\section{Processus de création dans le théâtre de marionnettes : présentation d'un dossier génétique}

Cet article présente de manière succincte le dossier génétique du Journal, spectacle du marionnettiste brésilien Álvaro Apocalypse, et montre toute la diversité des documents qui interviennent dans la création d'une telle œuvre, apparentée aux arts plastiques aussi bien qu'aux arts du texte et de la performance.

This article concisely presents the genetic dossier of the Journal, a puppet show of the Brazilian author Álvaro Apocalypse. It shows the great diversity of the documents that come into play in the creation of such a work related to plastic arts as much as to textual arts and performance art.

Dieser Artikel präsentiert in dichter Form das textgenetische Dossier von Journal, Theaterstück des brasilianischen Marionnettenspielers Àlvaro Apocalypse, und zeigt die gesamte Bandbreite der verschiedenen Dokumente, die an der Erschaffung eines solchen Werkes, das sowohl der Bildenden Kunst als auch den textuellen und performativen Künsten angehört, beteiligt sind.
Este artículo presenta de manera sucinta el dossier genético de Diario, espectáculo del titiritero brasileño Álvaro Apocalypse, mostrando la diversidad de documentos que intervienen en la creación de una obra de este tipo, vinculada tanto con las artes plásticas como con las artes del texto y de la performance.

$\mathbf{O}$ artigo apresenta de modo sucinto o dossier genético de Diário, espectáculo do marionetista brasileiro Álvaro Apocalypse, e mostra a diversidade de documentos que intervieram na criação da obra, que tem afinidades tanto com as artes plásticas quanto com as artes textuais e performativas.

Questo articolo presenta succintamente il fascicolo genetico del Journal, spettacolo del marionettista brasiliano Álvaro Apocalypse, e mostra tutta la diversità dei documenti che intervengono nella creazione di un'opera di questo genere, vicina sia alle arti plastiche sia alle arti del testo e dello spettacolo. 